\title{
Backstepping Global and Structural Stabilization of DC/DC Boost Converter
}

\author{
Zineb MADNI · Kamel GUESMI • Atallah BENALIA
}

Received: date / Accepted: date

\begin{abstract}
This paper deals with the stabilization of DC/DC boost converter and the nonlinear phenomena elimination using a constrained Backstepping technique. Based on the converter averaged model, the proposed control approach is designed and the input to state stability concept is used to proof the system global stability. Furthermore, the structural stability is proven to show the efficiency of the proposed approach to suppress the nonlinear phenomena exhibited by the converter. The simulation results illustrate the different regions of stability of the system and the bifurcation diagrams are given to show the effectiveness of the proposed approach in terms of nonlinear phenomena suppression.
\end{abstract}

Keywords Input to state stability · Global stability · Structural stability · Bifurcation · Boost converter · Backstepping · Switching frequency.

\section{Introduction}

In nowadays, the demand on energy is increasing and consumers claim for safe, clean and renewable resources like sun, wind and hydrogen. To this end, industries are developed around the PV panels, wind turbines, fuel cells, storage elements and other devices for optimal electricity production and storage. In all steps, from the electricity production to the deliverance to the end consumer, the converters play an important role in each phase of the process [29,30,33].

The objective of the converter is to shape the energy according to the demand using a switching mechanism governed by a control law. For years, many models and control techniques are proposed to analyze and to master the power converter complex behaviors. Indeed, many methods to analyze and control bifurcations and chaos in converters like Boost, Buck, Buck-Boost and other systems that suffer from these undesirable, complex and abnormal behaviors are reported in [2,13,17,37-39].

Zineb MADNI

LACoSERE, University of Laghouat, Algeria

E-mail: madnizineb@gmail.com

Kamel GUESMI

CReSTIC, University of Reims, France

E-mail: kamel.guesmi@univ-reims.fr

Atallah BENALIA

LACoSERE, University of Laghouat, Algeria

E-mail: a.benalia@lagh-univ.dz 
Researchers were mainly interested in the design of the adequate and the effective methods of analysis and control to achieve requirements like stability, performances and to deal, as well, with physical limitations of the converter elements. To this end, fuzzy logic based techniques are used in many works [8,23] and ensured satisfactory results. Nevertheless, fuzzy logic techniques need an accurate knowledge of the system behavior based on the human experience. On the other hand, the sliding mode based methods are used for the same task in $[21,28]$. However, the main drawback of such approaches is the chattering phenomenon. The predictive control approaches, with the inherent propriety of optimal prediction, can ensure better tracking of the reference as reported in [4,7]. The controllers based on state feedback are the most used for their simplicity and reduced cost of implementation $[11,20]$. When the performances of the aforementioned controllers are not satisfactory, the Backstepping can be a good alternative. Indeed, it presents the advantage to be a systematic design approach based on Lyapunov theory and it proved its effectiveness in many frameworks $[9,10,22,34]$.

Besides control performances, we are looking for effectiveness and ease of implementation. In this context, the digital controllers offer better results and are more efficient than the analog ones due to the rapid growth of the calculators' technology. With calculators, we can implement a digital controller directly or a discrete version of the continuous controller. In the case of switched power converters, the switching period must be the smallest that the setup can support to ensure performances with global asymptotic and/or exponential stability $[3,5,12,14,15,18,27,31,32,35,36]$. On the other hand, materials such $[1,6$, $16,19,24,26,37]$ studied the effect of the commutation frequency on the system qualitative behavior. It concluded, from the aforementioned materials, that the increase of the switching frequency is an efficient way to suppress nonlinear phenomena from the operating domain of the converter. However, imposing constraints on the switching period leads to a limited performance and enhancing this last, by increasing the switching frequency, can be an expensive solution or even impossible one due to the physical limitation of the switching elements.

To deal with this problem, we introduce in this paper some constraints on the controller parameters instead of those on the commutation frequency. Furthermore, we are interested by the plant structural stability due to the fact that global stability is not sufficient to ensure the same simple and predictable qualitative behavior of the system in the presence of disturbances. Besides the system global stability, our objective is to drive the system to work in a simple period one region and suppress all nonlinear phenomena such as bifurcation, chaos or other complex nonlinear behaviors. To this end, we developed a Backstepping controller based on the averaged model of the converter. The global stability analysis allowed us to obtain constraints on the controller parameters and the switching period. We keep constant the switching frequency and we play on the controller parameters to ensure the desired behavior of the system. Finally, the system structural stability is discussed as a continuity of the obtained asymptotic global stability.

Simulations are carried out to show the effectiveness of the proposed approach, to define the different regions of stability and to demonstrate the efficiency of the proposed controller to suppress undesired and abnormal behaviors of the converter.

The remains of this paper are given as follows: in the next section we present the converter model and the proposed Backstepping control law. In section 3, we analyze the whole system stability and in the last section we illustrate some simulation results that show the efficiency of the proposed approach and the obtained performances.

\section{Backstepping control of the Boost converter}

The Boost converter is depicted in figure 1. The block of Pulse Width Modulation (PWM) is omitted in this figure and supposed to be well-designed. 


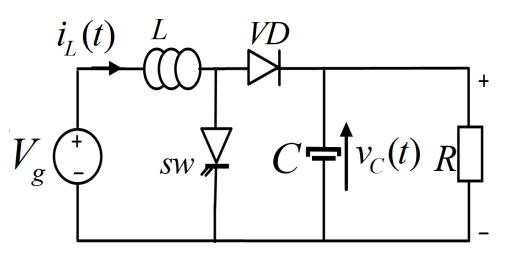

Fig. 1: Boost converter

The averaged dynamics of the converter, over a switching period $T$, are described by the model (1).

$$
\left\{\begin{array}{l}
\dot{x}_{1}=\frac{V_{g}}{L}-\frac{1}{L}(1-d) x_{2} \\
\dot{x}_{2}=\frac{(1-d)}{C} x_{1}-\frac{x_{2}}{R C}
\end{array}\right.
$$

with $x_{1}=<i_{L}>_{T}$ and $x_{2}=<v_{C}>_{T}$ the averaged values, over the switching period, of the inductance current and the capacitor voltage respectively.

To track a reference current $I_{r e f}$, we use the developed Backstepping control law:

$$
\dot{d}=\left[\left(c_{1}^{2}-(1-d)^{2}\right) e_{1}+\left(c_{1}+c_{2}\right)(1-d) e_{2}+\frac{(1-d)^{2}}{L C} x_{1}-\frac{(1-d)}{R L C} x_{2}+\frac{\ddot{I}_{r e f}}{(1-d)}\right] / \delta
$$

with $c_{1}, c_{2}$ positive parameters of the controller, $e_{1}=x_{1}-I_{r e f}$ the current error, $\frac{x_{2}}{L}$ the virtual control, $e_{2}=\frac{x_{2}}{L}-\delta$ the virtual control error and $\delta$ the objective function given by (3):

$$
\delta=\frac{1}{(1-d)}\left[c_{1} e_{1}+\frac{V_{g}}{L}-\dot{I}_{r e f}\right]
$$

The error dynamics are:

$$
\left\{\begin{array}{l}
\dot{e}_{1}=-c_{1} e_{1}-(1-d) e_{2} \\
\dot{e}_{2}=(1-d) e_{1}-c_{2} e_{2}
\end{array}\right.
$$

and the equations of the closed loop system are:

$$
\left\{\begin{array}{l}
\dot{e}=f(e, d) \\
\dot{d}=g(e, d)
\end{array}\right.
$$

where $\dot{e}=\left(\begin{array}{c}\dot{e}_{1} \\ \dot{e}_{2}\end{array}\right), g=\left(g_{1} e_{1}+g_{2} e_{2}+g_{3}\right) / \delta, g_{1}=\left(c_{1}^{2}-\frac{c_{1}}{R C}+\frac{(1-d)^{2}}{L C}-(1-d)^{2}\right), g_{2}=\left(c_{1}+c_{2}-\frac{1}{R C}\right)(1-$ $d), g_{3}=\left(\frac{(1-d)^{2}}{L C} I_{\text {ref }}-\frac{V_{g}}{R L C}\right)$

The discrete version of this model is:

$$
\begin{gathered}
\left\{\begin{array}{l}
\dot{e}=f(e(t), d) \\
\dot{d}=g(e(t), d)+e_{k}
\end{array}\right. \\
\left\{\begin{array}{l}
\dot{e}=f(e, d) \\
\dot{d}=g\left(e\left(t_{k}\right), d\right)
\end{array}\right.
\end{gathered}
$$

with $e_{k}$ the error between the continuous model and the discrete one, i.e.:

$$
e_{k}=g(e(t), d)-g\left(e\left(t_{k}\right), d\right)
$$




\section{Stability analysis}

3.1 Global stability

Before we analyze the system stability, let us introduce the following proprieties related to the main question:

1. The equilibrium point of the system is: $(e, d)=(0, D)$ with $0<\mathrm{d}<1$ that fluctuate around the value $D$ at steady state.

2. The system is input to state stable (ISS): $|e| \leq M_{1} \exp (-\theta t)+\gamma \sup _{0 \leq \tau \leq t}\left(\exp (-\theta(t-\tau))\left|e_{k}(\tau)\right|\right), \forall t \geq 0$, with $\theta=-\min \left(c_{1}, c_{2}\right), M_{1}=\sqrt{2 V(t=0)}, \gamma$ a positive constant and $V($.$) the Lyapunov function.$

3. The system dynamics are bounded: $f(0, d)=\left(\begin{array}{l}0 \\ 0\end{array}\right),|f(e, d)| \leq \beta|e|$ with $\beta=\sqrt{c_{1}^{2}+c_{2}^{2}+1+\left|c_{1}-c_{2}\right|}$

4. The system is Lipschitz: $|f(e, d)-f(y, d)| \leq \beta|e-y|$.

$$
|g(e, d)-g(y, d)| \leq N|e-y|
$$

with $e=\left(\begin{array}{l}e_{1} \\ e_{2}\end{array}\right)$ and $y=\left(\begin{array}{l}y_{1} \\ y_{2}\end{array}\right)$ two different values of the error, $N=\delta_{1 \max } g_{12}+c_{1} \delta_{2 \max } \delta_{1 \text { max }}^{2}$ with $\delta_{1 \max }, \delta_{2 \max }, g_{12}$ positive parameters defined in the appendix.

Proofs of the above proprieties are given in the appendix.

Based on these proprieties, we can announce the following theorem:

Theorem 1 For the converter given by equation (6), the system is globally asymptotically stable if the following constraints are verified:

1. $e_{1}(0)=-I_{\text {ref }}$ (null initial condition)

2. $c_{1}<\frac{V_{g}}{L I_{r e f}}$

3. $\beta \gamma N<\frac{1}{T}$

Proof The first condition means that the inductance is initially discharged which is a trivial condition and easy to verify. The error is bounded starting from $e_{1}(0)=-I_{r e f}$ and converges exponentially to zero according to the ISS propriety. Indeed, $-I_{r e f} \leq e_{1}$ means that: $-c_{1} I_{r e f}+\frac{V_{g}}{L} \leq c_{1} e_{1}+\frac{V_{g}}{L}$

From equations (2) and (3) with $0<d<1$ and the positive objective function, we have:

$\left(c_{1} e_{1}+\frac{V_{g}}{L}\right)>0, \forall e_{1} \leq 0$

Leading to:

$0<\frac{1}{c_{1} e_{1}+\frac{V_{g}}{L}} \leq \frac{1}{-c_{1} I_{r e f}+\frac{V_{g}}{L}}$

So, $c_{1}<\frac{V_{g}}{L I_{\text {ref }}}$

Hence, for the last constraint, the system is ISS and has the form:

$$
e(t) \leq M_{1} \exp (-\theta t)+\gamma \sup _{0 \leq \tau \leq t}(\exp (-\theta(t-\tau)))\left|e_{k}(\tau)\right|
$$

and the global asymptotic stability will be proven as follows:

From equations (7) and (8) we have:

$$
\left|e_{k}(t)\right| \leq N\left|e(t)-e\left(t_{k}\right)\right|
$$

Using (9) and (10) we obtain:

$$
|e(t)| \leq M_{1} \exp (-\theta t)+\gamma N \sup _{0 \leq \tau \leq t}\left[\exp (-\theta(t-\tau))\left|e(\tau)-e\left(t_{k}\right)\right|\right]
$$




$$
\exp (\theta t)|e(t)| \leq M_{1}+\gamma N \sup _{0 \leq \tau \leq t}\left[\exp (\theta \tau)\left|e(\tau)-e\left(t_{k}\right)\right|\right]
$$

We have:

$$
\begin{gathered}
e(t)=e\left(t_{k}\right)+\int_{t_{k}}^{t} f(e(\tau), d) d \tau \\
\left.e(t)-e\left(t_{k}\right)=\int_{t_{k}}^{t}\left(f(e(\tau), d)-f\left(e\left(t_{k}\right), d\right)\right) d \tau+\int_{t_{k}}^{t} f\left(e\left(t_{k}\right), d\right)\right) d \tau \\
\left|e(t)-e\left(t_{k}\right)\right| \leq \int_{t_{k}}^{t}\left|f(e(\tau), d)-f\left(e\left(t_{k}\right), d\right)\right| d \tau+\int_{t_{k}}^{t}\left|f\left(e\left(t_{k}\right), d\right)\right| d \tau \\
\left|e(t)-e\left(t_{k}\right)\right| \leq \beta \int_{t_{k}}^{t}\left|e(\tau)-e\left(t_{k}\right)\right| d \tau+\beta T\left|e\left(t_{k}\right)\right|
\end{gathered}
$$

and using Gronwall-Bellman Lemma [25] we obtain:

$$
\left|e(t)-e\left(t_{k}\right)\right| \leq \beta T \exp (\beta T)\left|e\left(t_{k}\right)\right|
$$

Leading to:

$$
\left|e(t)-e\left(t_{k}\right)\right| \leq \beta T \exp (\beta T)\left|e\left(t_{k}\right)-e(t)+e(t)\right|
$$

So,

$$
(1-\beta T \exp (\beta T))\left|e\left(t_{k}\right)-e(t)\right| \leq \beta T \exp (\beta T)|e(t)|
$$

If $(1-\beta T \exp (\beta T))>0$ then:

$$
\left|e\left(t_{k}\right)-e(t)\right| \leq \frac{\beta T \exp (\beta T)}{(1-\beta T \exp (\beta T))}|e(t)|
$$

and

$$
\sup _{0 \leq \tau \leq t} \exp (\theta \tau)\left|e\left(t_{k}\right)-e(\tau)\right| \leq \sup _{0 \leq \tau \leq t}\left[\frac{\beta T \exp (\beta T)}{(1-\beta T \exp (\beta T))} \exp (\theta \tau)|e(\tau)|\right]
$$

From (12) and (15) we have:

$$
\begin{gathered}
\exp (\theta t)|e(t)| \leq M_{1}+\gamma N \frac{\beta T \exp (\beta T)}{(1-\beta T \exp (\beta T))} \sup _{0 \leq \tau \leq t} \exp (\theta \tau)|e(\tau)| \\
\exp (\theta t)|e(t)| \leq M_{1}+M_{2} \sup _{0 \leq \tau \leq t} \exp (\theta \tau)|e(\tau)|
\end{gathered}
$$

with: $M_{2}=\frac{\gamma N \beta T \exp (\beta T)}{(1-\beta T \exp (\beta T))}$

So,

$$
\sup _{0 \leq \tau \leq t} \exp (\theta \tau)|e(\tau)| \leq M_{1}+M_{2} \sup _{0 \leq \tau \leq t} \exp (\theta \tau)|e(\tau)|
$$

Hence,

$$
\left(1-M_{2}\right) \sup _{0 \leq \tau \leq t} \exp (\theta \tau)|e(\tau)| \leq M_{1}
$$

If $\left(1-M_{2}\right)>0$ then $\sup _{0 \leq \tau \leq t} \exp (\theta \tau)|e(\tau)| \leq \frac{M_{1}}{1-M_{2}}$

Which mean $|e(t)| \leq \frac{M_{1}}{\left(1-M_{2}\right)} \exp (-\theta t)$ and

$$
\lim _{t \rightarrow \infty}|e(t)|=0
$$


Hence, the global asymptotic stability is verified when the following conditions are verified:

$$
\begin{gathered}
1-\beta T \exp (\beta T)>0 \\
1-M_{2}>0
\end{gathered}
$$

With the fact that $\beta T>0, \exp (\beta T)>1$, the inequalities (17) and (18) can be merged in the following unique condition:

$$
\beta \gamma N<\frac{1}{T}
$$

\subsection{Structural stability}

To study the closed loop system structural stability, let us illustrate the loop portion containing the PWM block depicted in figure 2. The controller followed by the saturation block deliver, at the instant $k T$, a value of the duty cycle within the interval $d \in] 0,1\left[\right.$. This last will be kept constant during the actual $\left(k^{t h}\right)$ commutation period and compared to sawtooth signal to generate the OFF switching signal while the clock pulse generates the ON signal. The latch $R S$ helps on obtaining a unique commutation during each switching period. A well-designed PWM generates one commutation signal by switching period.

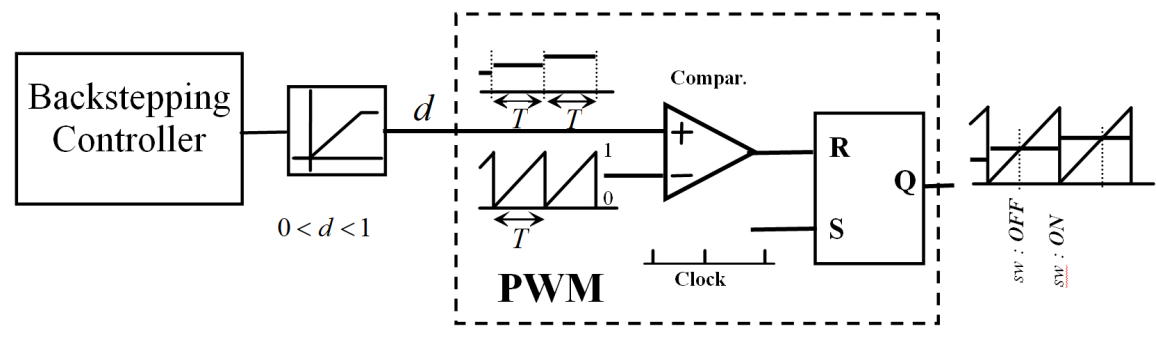

Fig. 2: Controller with PWM block

Proposition: The proposed Backstepping controller ensures the system structural stability if:

1. The controller parameters $c_{1}, c_{2}$ are chosen within the stable zones

2. The PWM is well-designed to ensure only one commutation by switching period

Proof If we suppose that the system attain the reference current at the instant $t=k T$, the $1^{\text {st }}$ condition means the asymptotic stability of the system; hence,

$$
<i_{L}>_{(k T)}=<i_{L}>_{((k+1) T)}=I_{r e f}
$$

On the other hand, the unique commutation during each switching period means that we have unique cycle of inductance charge-discharge during a switching period with the same averaged value; hence,

$$
i_{L}(k T)=i_{L}((k+1) T)
$$

This invariance of the converter dynamical behavior means that the same cycle of inductance chargedischarge is repeated during the steady state which proofs the structural stability of the system. 


\section{Simulation results}

In order to show the efficiency of the proposed approach and to illustrate the ensured global and structural stabilities, we use the converter parameters given in table 1 .

\begin{tabular}{cc}
\hline Parameter & Value \\
\hline Input voltage (Vg) & $15 V$ \\
Load (R) & $30 \Omega$ \\
Inductance (L) & $20.10^{-3} \mathrm{H}$ \\
Capacitor (C) & $68.10^{-6} \mathrm{~F}$ \\
\hline
\end{tabular}

Table 1: Converter parameters

Next, we use the proposed approach to define the stability regions, we validate the proposed controller and we show its efficiency to ensure the system global and structural stabilities.

4.1 Global stability regions

In what follows, we explore the plan searching for global stability regions of the system. The obtained results are depicted in figure 3 .

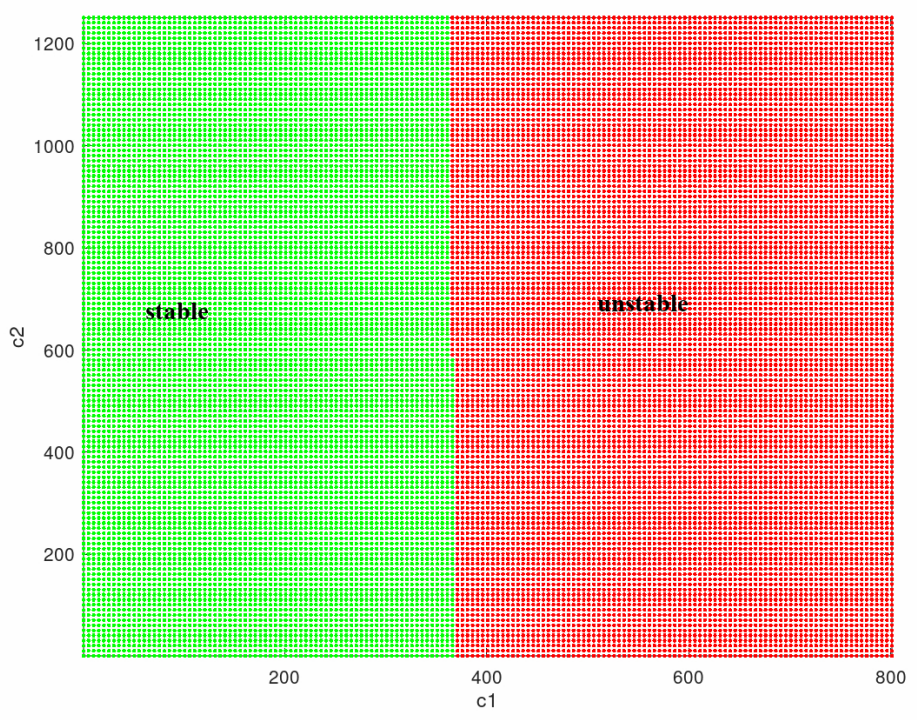

Fig. 3: Stability region $\left(c_{1}, c_{2}\right)$

From figure 3 we remark the slight effect of the parameter $c_{2}$ on the system global stability. Indeed, this fact is expected from the $3^{r d}$ constraint of the theorem where for high frequencies the right term tends 
practically to infinity and any value of $c_{2}$ verifies this constraint. However, the parameter $c_{1}$ must verify the $2^{\text {nd }}$ constraint of the theorem; that depends on the system parameters $\left(V_{g}\right.$ and $\left.L\right)$ and the reference current. Hence, we can define the system stability region based only on $c_{1}$ and this fact reduces considerably the complexity of the study. From practical point of view and as mentioned in the theorem, the system global stability depends also on the switching frequency, the reference current and the load value. Figures 4,5 and 6 summarize the effect of these parameters on the system stability.

Figure 4 shows clearly the fact that the increasing of the switching frequency leads to enlarging the

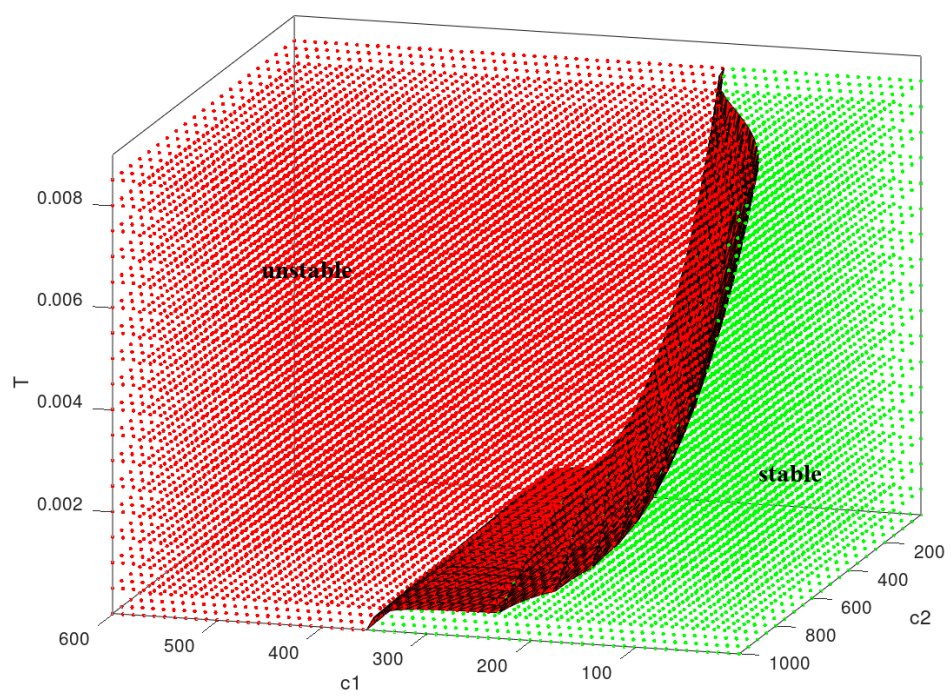

Fig. 4: Stability region $\left(c_{1}, c_{2}, T\right)$

stability zone. However, we should mention that for practical convince we should increase the frequency till we approach the switch $s w$ nominal frequency; after that we decrease the parameter $c_{1}$ value to obtain the wider region of stability.

Figure 5 confirms the $2^{\text {nd }}$ constraint of the theorem. Indeed, it shows that the increase of the reference current leads to the shrinking of the stability zone. Hence, to ensure the system stability, the level of energy to attain by the converter should stay in a reasonable interval. The parameter $c_{1}$, then can be used to enlarge the stability zone and to ensure that the converter operates in a safe stable zone.

From figure 6 we state that the effect of the load on the system stability is quite linear and the increasing of the load value leads to a larger stability zone and a larger interval for a safe choice of the parameter $c_{1}$ value. This fact can be physically understood due to the fact that for important values of the load, the system is more dissipative and consequently more stable. However, for slight load, the stability zone is reduced and for stability requirement the $c_{1}$ must take small values. 


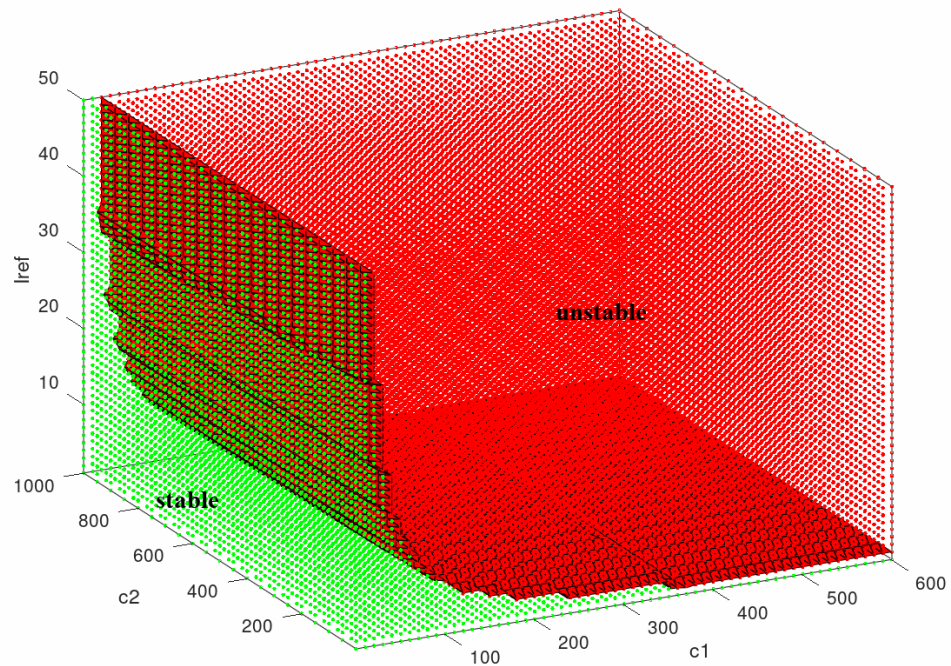

Fig. 5: Stability region $\left(c_{1}, c_{2}, I_{r e f}\right)$

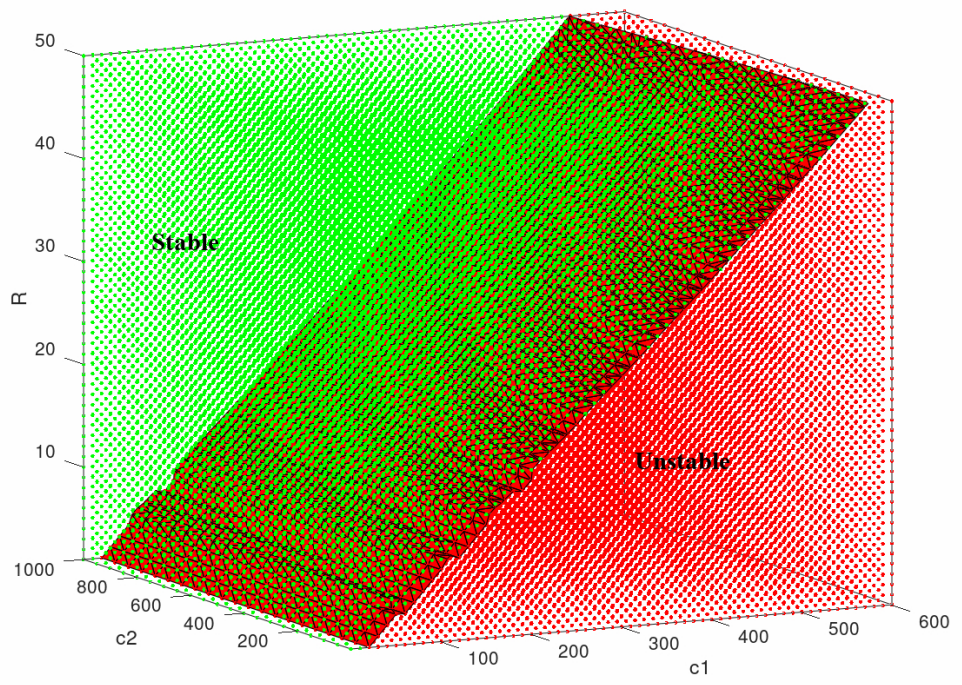

Fig. 6: Stability region $\left(c_{1}, c_{2}, R\right)$

4.2 Nonlinear phenomena elimination

To study the nonlinear phenomena exhibited by the converter we use the bifurcation diagram as analysis tool. The abnormal behaviors of the converter are obtained using the following feedback control law:

$$
d=\frac{L}{T}\left(\frac{I_{r e f}-i_{L}}{V_{g}}\right)
$$


At first and to show the effect of switching frequency on structural stability we illustrate the bifurcation diagrams for two values of the switching frequency and for both cases of the bifurcation parameters; reference current and load.

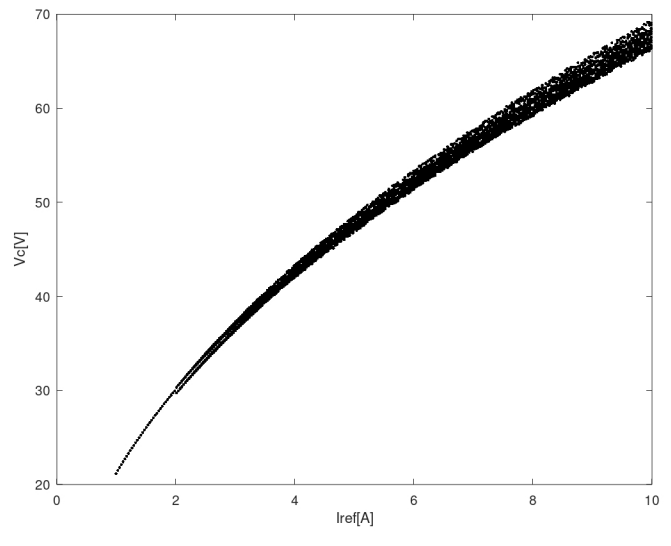

(a) $T=5 \cdot 10^{-5}$

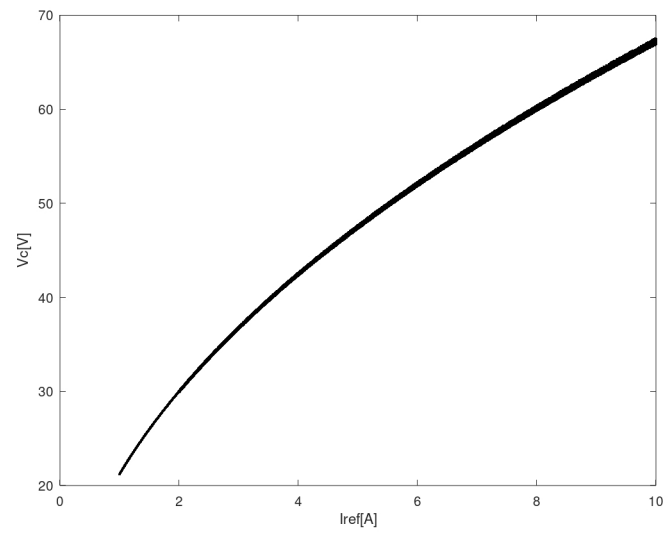

(b) $T=10^{-5}$

Fig. 7: Abnormal behaviors varying $I_{\text {ref }}$

Figure 7a shows that the system has a simple period-one (1T) behavior till the value $1.98 \mathrm{~A}$ of the reference current; beyond this value the system exhibits period doubling bifurcation and chaos for important values of $I_{r e f}$. To solve such problem, we can increase the switching frequency to force the system to have a simple predictable $1 \mathrm{~T}$ behavior. Indeed, if we increase five times the switching frequency, we state from figure $7 \mathrm{~b}$ the enhancement introduced on the system behavior and the total suppression of the complex nonlinear phenomena. Similarly, the original behavior in the case of load variation (figure 8a) contains $1 \mathrm{~T}$ behavior for slight loads till the value $R=30 \Omega$ and beyond this value the system behavior becomes more and more complicated until it reaches the chaos zone. By increasing the switching frequency the system behavior becomes simpler and predictable and the zone of $1 \mathrm{~T}$ behavior becomes wider and occupy the whole range as shown in figure $8 \mathrm{~b}$.

Figures 7 and 8 confirm the fact that the switching frequency increase can be an effective solution to suppress abnormal, complex and undesirable phenomena from the converter behavior; however, this increases stress on the switching element and is an expensive solution or even impossible one for high frequencies requirement and due to the physical limitation of the commutation element.

The proposed solution was to ensure the same performances and stability using, instead, the controller parameters $\left(c_{1}, c_{2}\right)$ while keeping the switching frequency in reasonable range to avoid the aforementioned issues. Indeed, if we choose $c_{1}=70, c_{2}=900$ and we fix the switching period at $T=5.10^{-5}$, the obtained results are given in figures 9 and 10 for varying $I_{\text {ref }}$ and $R$ respectively.

In the case of varying $I_{r e f}$ and comparing the proposed controller result of figure $9 \mathrm{~b}$ to the original behavior of figure $9 \mathrm{a}$ and to the switching frequency based enhancement of figure 7b, we remark that the proposed Backstepping ensures the simpler $1 \mathrm{~T}$ behavior on the whole range without frequency constraints on the switch. Indeed, even the proposed controller (Fig. 9b) uses the base switching frequency $\left(T=5 \cdot 10^{-5}\right)$, it ensures better results than those obtained with increased switching frequency solution (Fig. 7b). Finally, when the load is subject to variation, we remark from figure 10b that the proposed controller, operating with the base switching frequency $\left(T=5 \cdot 10^{-5}\right)$, enhances considerably the original behavior depicted in 


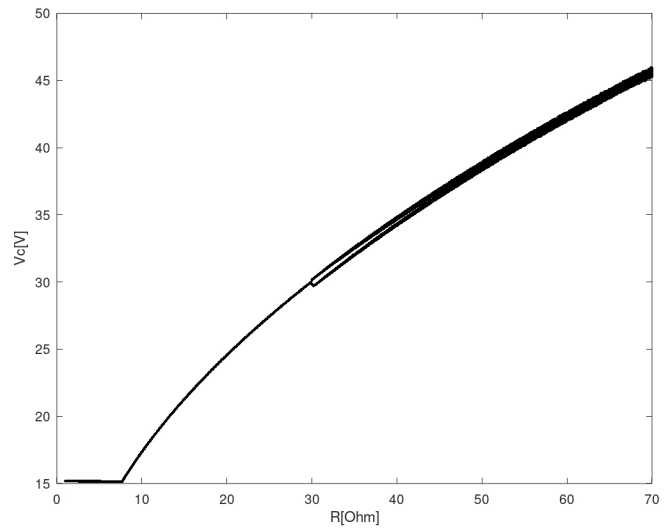

(a) $T=5 \cdot 10^{-5}$

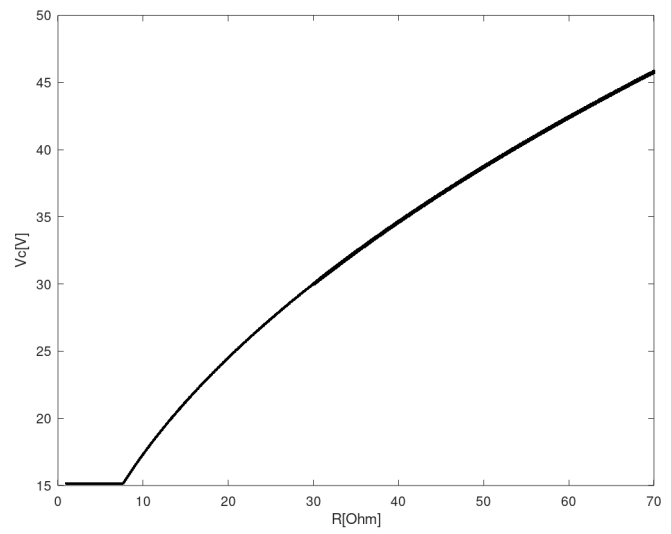

(b) $T=10^{-5}$

Fig. 8: Abnormal behaviors varying $R$

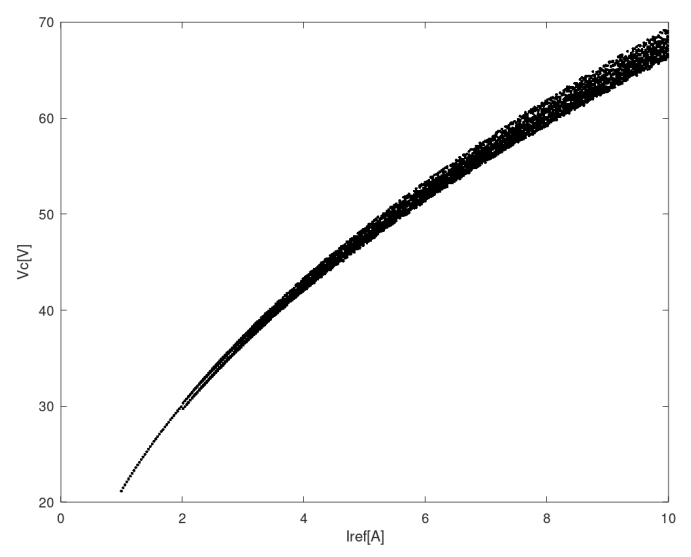

(a) Original

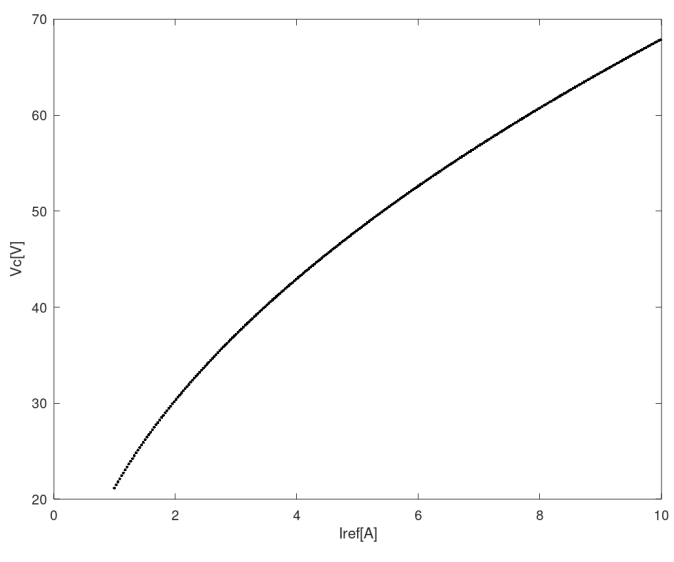

(b) Enhanced using Backstepping

Fig. 9: Enhanced and original behaviors varying $I_{\text {ref }},\left(c_{1}=70, c_{2}=900, T=5.10^{-5}\right)$

figure 10a and ensures better performance and simpler behavior than the one ensured by the solution based on increasing the switching frequency (Fig. 8b).

\section{Conclusion}

In this paper, we developed a Backstepping control law for a DC/DC Boost converter. Besides the control performance, the global stability of the closed loop system is proven as well as the structural stability. We showed, on the one hand, that the system stability is mainly related to one parameter of the controller which simplifies, considerably, the control and the stabilization tasks. On the other hand, the proposed approach ensured the total suppression of abnormal and undesired phenomena from the operating domain of the 


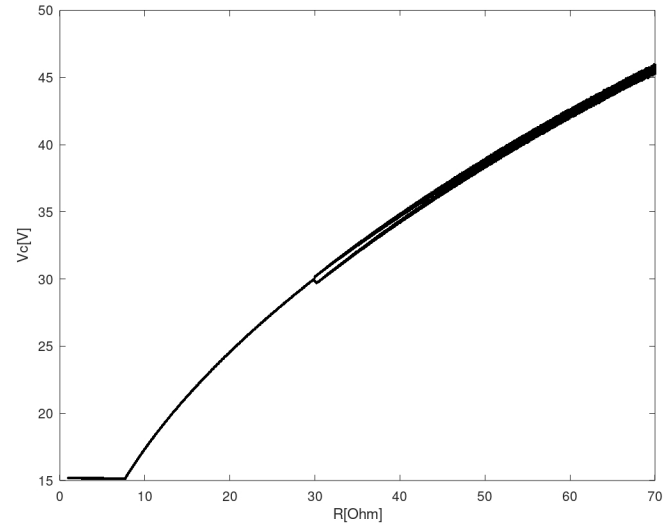

(a) Original

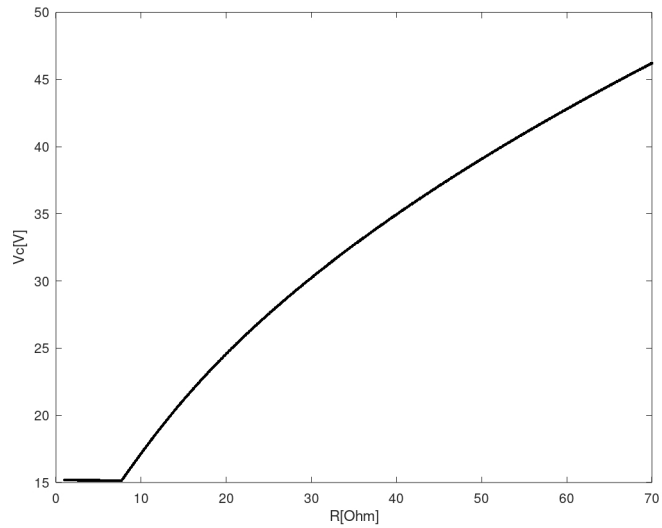

(b) Enhanced using Backstepping

Fig. 10: Enhanced and original behaviors varying $R,\left(c_{1}=70, c_{2}=900, T=5.10^{-5}\right)$

converter instead of increasing the switching frequency. Simulation results confirmed the different theoretical conclusions and showed the efficiency of the proposed approach to stabilize globally asymptotically the system and to ensure a simple forcastable period-one behavior of the system on the whole operating domain.

\section{A Appendix}

\section{A.1 Equilibrium point}

The equilibrium point satisfying $(\dot{e}, \dot{d})=(0,0)$ is: $(e, d)=(0, D)$

\section{A.2 Input to state stability}

The Lyapunov candidate function is: $V=\left(e_{1}^{2}+e_{2}^{2}\right) / 2$ and its time derivative is: $\dot{V}=-c_{1} e_{1}^{2}-c_{2} e_{2}^{2}$, hence:

$$
\begin{gathered}
\dot{V} \leq-\min \left(c_{1}, c_{2}\right)\left(e_{1}^{2}+e_{2}^{2}\right) \\
\dot{V} \leq-2 \min \left(c_{1}, c_{2}\right) V
\end{gathered}
$$

and we can obtain:

$$
\begin{gathered}
V \leq V(t=0) \exp \left(-2 \min \left(c_{1}, c_{2}\right) t\right) \\
|e|=\sqrt{2 V} \\
|e| \leq \sqrt{2 V(t=0)} \exp \left(-\min \left(c_{1}, c_{2}\right) t\right)
\end{gathered}
$$

This expression has the form:

$$
|e| \leq M_{1} \exp (-\theta t)+\gamma \sup _{0 \leq \tau \leq t}\left(\exp (-\theta(t-\tau))\left|e_{k}(\tau)\right|\right), \forall t \geq 0
$$

where: $\theta=-\min \left(c_{1}, c_{2}\right), M_{1}=\sqrt{2 V(t=0)}, \gamma=0$. which confirms that the system is input to state stable. 


\section{A.3 Boundness}

We have: $f(0, d)=\left(\begin{array}{l}0 \\ 0\end{array}\right)$,

$|f(e, d)|=\sqrt{\left(c_{1}^{2}+(1-d)^{2}\right) e_{1}^{2}+\left(c_{2}^{2}+(1-d)^{2}\right) e_{2}^{2}+2 e_{1} e_{2}(1-d)\left(c_{1}-c_{2}\right)}$

With the fact that: $2 e_{1} e_{2} \leq\left(e_{1}^{2}+e_{2}^{2}\right)$,

$$
(1-d)\left(c_{1}-c_{2}\right) \leq\left|c_{1}-c_{2}\right|
$$

and

$$
\begin{aligned}
& \left(c_{1}^{2}+(1-d)^{2}\right) \leq\left(c_{1}^{2}+1\right) \\
& \left(c_{2}^{2}+(1-d)^{2}\right) \leq\left(c_{2}^{2}+1\right)
\end{aligned}
$$

we can obtain

$$
\begin{gathered}
|f(e, d)| \leq \sqrt{\left(c_{1}^{2} e_{1}^{2}+c_{2}^{2} e_{2}^{2}\right)+\left(e_{1}^{2}+e_{2}^{2}\right)+\left(e_{1}^{2}+e_{2}^{2}\right)\left|c_{1}-c_{2}\right|} \\
|f(e, d)| \leq \sqrt{\left(c_{1}^{2}+c_{2}^{2}+1+\left|c_{1}-c_{2}\right|\right) e^{2}}
\end{gathered}
$$

Hence,

$$
|f(e, d)| \leq \beta|e|
$$

\section{A.4 Lipschitz property}

For the error system, we have:

$$
\begin{gathered}
f(e, d)-f(y, d)=\left(\begin{array}{c}
-c_{1} e_{1}-(1-d) e_{2}+c_{1} y_{1}+(1-d) y_{2} \\
(1-d) e_{1}-c_{2} e_{2}-(1-d) y_{1}+c_{2} y_{2}
\end{array}\right) \\
|f(e, d)-f(y, d)|=\sqrt{\left(-c_{1}\left(e_{1}-y_{1}\right)-(1-d)\left(e_{2}-y_{2}\right)\right)^{2}+\left((1-d)\left(e_{1}-y_{1}\right)-c_{2}\left(e_{2}-y_{2}\right)\right)^{2}}
\end{gathered}
$$

Using the inequalities (A.1), (A.2) and (A.3) to obtain:

$$
\begin{gathered}
|f(e, d)-f(y, d)| \leq \sqrt{\left(c_{1}^{2}+1\right)\left(e_{1}-y_{1}\right)^{2}+\left(c_{2}^{2}+1\right)\left(e_{2}-y_{2}\right)^{2}+2\left(e_{1}-y_{1}\right)\left(e_{2}-y_{2}\right)\left|c_{1}-c_{2}\right|} \\
|f(e, d)-f(y, d)| \leq \sqrt{\left(c_{1}^{2}+c_{2}^{2}+1+\left|c_{1}-c_{2}\right|\right)\left(\left(e_{1}-y_{1}\right)^{2}+\left(e_{2}-y_{2}\right)^{2}\right)}
\end{gathered}
$$

Hence,

$$
|f(e, d)-f(y, d)| \leq \beta|e-y|
$$

which confirms the Lipschitz property of the error system.

For the control law, we have: $g(e, d)=\delta_{1}(e) \delta_{2}(e)$ with $\delta_{1}(e)=\frac{(1-d)}{c_{1} e_{1}+V_{g} / L}$ and $\delta_{2}(e)=g_{1} e_{1}+g_{2} e_{2}+g_{3}$ allows obtaining:

$$
\begin{gathered}
|g(e, d)-g(y, d)|=\left|\delta_{1}(e)\left(\delta_{2}(e)-\delta_{2}(y)\right)+\delta_{2}(y)\left(\delta_{1}(e)-\delta_{1}(y)\right)\right| \\
|g(e, d)-g(y, d)| \leq\left|\delta_{1}(e)\right|\left|\left(\delta_{2}(e)-\delta_{2}(y)\right)\right|+\left|\delta_{2}(y)\right|\left|\delta_{1}(e)-\delta_{1}(y)\right|
\end{gathered}
$$

$\delta_{1} \leq \delta_{1 \max }$ and $\left|\delta_{2}(e)-\delta_{2}(y)\right| \leq g_{12}|(e-y)|$ with $g_{12}=\left|g_{1 \max }\right|+\left|g_{2 \max }\right|$

and $\left|\delta_{2}\right| \leq \delta_{2 \max }$ with $\delta_{2 \max }=g_{1 \max } e_{1 \max }+g_{2 \max } e_{2 \max }+g_{3 \max }$

$$
\left|\delta_{1}(e)-\delta_{1}(y)\right| \leq c_{1} \delta_{1 \max }^{2}|e-y|
$$

Hence,

$$
|g(e, d)-g(y, d)| \leq N|e-y|, N=\delta_{1 \max } g_{12}+c_{1} \delta_{2 \max } \delta_{1 \max }^{2}
$$

This concludes the proof of the Lipschitz propriety of both error system and control law. 
A.5 Maximum values calculation

$\delta_{1} \max =?$

Since $0<d<1$, we can write:

$$
\delta_{1}<\frac{1}{c_{1} e_{1}+V_{g} / L} \leq \delta_{1 \max }
$$

we shown that the error is bounded, has an exponential form and vanish from its maximum value (the initial value). If we consider that the system is initially uncharged $\left(e_{1}(0) \leq-I_{r e f}\right)$ then $-c_{1} I_{r e f}+V_{g} / L \leq c_{1} e_{1}+V_{g} / L$. The positiveness of the objective function $\delta=\frac{1}{(1-d)}\left(c_{1} e_{1}-\dot{I}_{\text {ref }}+V_{g} / L\right)$ with constant reference current leads to:

$$
\delta_{1 \max }=\frac{1}{-c_{1} I_{\text {ref }}+V_{g} / L}
$$

$\delta_{2} \max =?$

Similarly to the previous calculation, we can write:

$$
-(1-d)^{2}<\frac{(1-d)^{2}}{L C}-(1-d)^{2}<\frac{1}{L C}-(1-d)^{2}
$$

and

Hence,

$$
-1+\frac{1}{L C}<\frac{1}{L C}-(1-d)^{2}<\frac{1}{L C}
$$

$$
g_{1 \max }=\left|c_{1}^{2}-\frac{c_{1}}{R C}+\frac{1}{L C}\right|
$$

We have also $0<\left(c_{1}+c_{2}\right)(1-d)<\left(c_{1}+c_{2}\right)$ and $\frac{-1}{R C}<-\frac{1}{R C}(1-d)<0$ which give:

$$
g_{2 \max }=c_{1}+c_{2}
$$

The fact that $0<d<1$, allows obtaining:

$$
g_{3 \max }=\frac{1}{L C} I_{\text {ref }}-\frac{V_{g}}{R L C}
$$

and finally,

$$
\delta_{2 \max }=g_{1 \max } e_{1 \max }+g_{2 \max } e_{2 \max }+g_{3 \max }
$$

\section{Conflict of interest}

The authors declare that they have no conflict of interest.

\section{Data availability statement}

All data generated or analyzed during this study are included in this published article.

\section{References}

1. Baja, M., Cormerais, H., Buisson, J.: Stabilizing control with commutation frequency limitations for a step-up converter. In: IFAC Conference on Management and Control of Production and Logistics (2007)

2. Bao, B., Zhang, X., Bao, H., Wu, P., Wu, Z., Chen, M.: Dynamical effects of memristive load on peak current mode Buck-Boost switching converter. Chaos, Solitons \& Fractals 122, 69-79 (2019)

3. Cao, Z., Gao, L., Wang, G.: Stability conditions of switched nonlinear systems with unstable subsystems and destabilizing switching behaviors. Applied Mathematics and Computation 363, 124558 (2019)

4. Cavanini, L., Cimini, G., Ippoliti, G.: Model predictive control for pre-compensated power converters: Application to current mode control. Journal of the Franklin Institute 356, 2015-2030 (2019) 
5. Chen, G., Deng, F., Yang, Y.: Practical finite time sability of switch nonlinear time systems based on initial state. Nonlinear Analysis: Hybrid systems 41, 101031 (2021)

6. Coronado-Mendoza, A., Gurubel-Tun, K.J., Zuniga-Grajeda, V., Domínguez-Navarro, J.A., Artal-Sevil, J.S.: Variable frequency control of a photovoltaic Boost converter system with power quality indexes based on dynamic phasors. In: IFAC PapersOnLine, p. 180-185 (2018)

7. Cunha, R.B.A., Inomoto, R.S., Altuna, J.A.T., Costa, F.F., DiSanto, S.G., Filho, A.J.S.: Constant switching frequency finite control set model predictive control applied to the Boost converter of a photovoltaic system. Solar Energy 189, 57-66 (2019)

8. Dorji, S., D.Wangchuk, Choden, T., Tshewang, T.: Maximum power point tracking of photovoltaic cell using Perturb \& Observe and fuzzy logic controller algorithm for Boost converter and quadratic Boost converter. Materials Today: Proceedings 27, 1224-1229 (2020)

9. Doubabi, H., Salhi, I., M.Chennani, Essounbouli, N.: High performance MPPT based on TS fuzzy-integral backstepping control for PV system under rapid varying irradiance-experimental validation. ISA Transactions 21 (2021)

10. Fadil, H.E., Giri, F., Ahmed-Ali, T., Gaouzi, K.: Stability analysis of nonlinear digital control of DC-DC Boost power converters with dynamical feedback. In: IFAC-PapersOnLine, p. 211-216 (2016)

11. Gkizas, G., Yfoulis, C., Amanatidis, C., Stergiopoulos, F., Giaouris, D., Ziogou, C., Voutetakis, S., Papadopoulou, S.: Digital state-feedback control of an interleaved DC/DC Boost converter with bifurcation analysis. Control Engineering Practice 73, 100-111 (2018)

12. Kivilcim, A., Karabacak, O., Wiesnieski, R.: Almost global stability of nonlinear switched systems with mode-dependent an edge-dependent average dwell time. Nonlinear Analysis: Hybrid systems 41, 101052 (2021)

13. Kumar, P., Narayanan, S.: Chaos and bifurcation analysis of stochastically excited discontinuous nonlinear oscillators. Nonlinear Dynamics 102, 927-950 (2020)

14. Kundu, A.: A new condition for stability of switched linear systems under restricted minimum dwell time switching. Systems \& Control Letters 135, 104597 (2020)

15. Kundu, A., Chatterjee, D.: Robust matrix commutator conditions for stability of switched linear systems under restricted switching. Automatica 115, 108904 (2020)

16. Li, Q., Chen, J., Jiang, D.: Periodic variation in the effect of switching frequency on the harmonics of power electronic converters. Chinese Journal of Electrical Engineerings 6 (2020)

17. Li, S., Fahimi, B., Kiani, M.: Bifurcation analysis and experimentation of peak current-controlled Boost converter. In: Annual Conference of the IEEE Industrial Electronics Society, pp. 1633-1638 (2019)

18. Li, S., Xiang, Z., Zhangn, J.: Exponential stability analysis for singular switched positive systems under dwell-time constraints. Journal of the Franklin Institute 357, 13834-13871 (2020)

19. Lin, X., Li, X., Chen, C.C., Shihua, L.: Global stabilization of switched nonlinear systems under arbitrary switchings via smooth output feedback. In: Chinese Control Conference (2017)

20. Liu, G., Zhang, X.: State feedback control and applied to PV systems under improved MPPT control. In: IEEE International Conference on Control Science and Systems Engineering (2018)

21. Mehta, A., Naik, B.: Sliding Mode Controller with PI-Type Sliding Function for DC/DC Buck Converter. Springer Nature Singapore Pte Ltd. (2019)

22. Mourabit, E.Y., Derouich, A., Ghzizal, A.E., Bouchnaif, J., Ouanjli, N.E., Zamzoum, O., Mezioui, K., Bossoufi, B.: Implementation and validation of backstepping control for PMSG wind turbine using dSPACE controller board. Energy Reports 5, 807-821 (2019)

23. Moustafa, E., Sobaih, A., Abozalam, B., Sayed, A., Mahmoud, A.: Period doubling bifurcation analysis and chaos control for load torque using FLC. Complex \& Intelligent Systems 7, 1381-1389 (2021)

24. Nandankar, P.V., Bedekar, P.P., Dahwas, P.K.V.: Variable switching frequency control for efficient DC/DC converter. Material Today: Proceedings (2021)

25. N'Doye, I., Zasadzinski, M., Darouach, M., Radhy, N.E., Bouaziz, A.: Exponential stabilization of a class of nonlinear systems: A generalized Gronwall-Bellman lemma approach. Nonlinear Analysis 74, 7333-7341 (2011)

26. Pai, M.A., Sauer, P.W., Lesieutre, B.C., Adapa, R.: Structural stability in power systems-effect of load models. IEEE Transactions on Power Systems 10, 609-615 (1995)

27. Qi, J., Sun, Y.: Global exponential stability of certain switched systems with time-varying delays. Applied Mathematics Letters 26, 760-765 (2013)

28. Qi, Q., Ghaderi, D., Guerrero, J.M.: Sliding mode controller-based switched-capacitor-based high DC gain and low voltage stress DC/DC Boost converter for photovoltaic applications. Electrical Power and Energy Systems 125, 1-19 (2021)

29. Salimi, M.: Practical implementation of the Lyapunov based nonlinear controller in DC/DC Boost converter for MPPT of the PV systems. Solar Energy 173, 246-255 (2018)

30. Spiliotis, K., Goncalves, J.E., DeSande, W.V., Ravyts, S., Daenen, M., Saelens, D., Baert, K., Driesen, J.: Modeling and validation of a DC/DC power converter for building energy simulations: Application to BIPV systems. Applied Energy 240, 646-665 (2019)

31. Sun, T., Liu, T., Sun, X.: Stability analysis of cyclic switched linear systems: An average cycle dwell time approach. Information Sciences 544, 227-237 (2021) 
32. Wang, G., Liu, Y., Lu, J., Wang, Z.: Stability analysis of totally positive switched linear systems with average dwell time switching. Nonlinear Analysis: Hybrid Systems 36, 100877 (2020)

33. Wu, Y., Huangfu, Y., Ma, R., Ravey, A., Chrenko, D.: A strong robust DC/DC converter of all-digital high-order sliding mode control for fuel cell power applications. Journal of Power Sources 413, 222-232 (2019)

34. Yin, X., Zhang, W., Jiang, Z., Pand, L., Lei, M.: Adaptive backstepping control for maximizing marine current power generation based on uncertainty and disturbance estimation. Electrical Power and Energy Systems 117, 105329 (2020)

35. Yu, Q., Lv, H.: The new stability criteria of discrete-time switched systems with an improved mode dependent average dwell time approach. Applied Mathematics and Computation 366, 124730 (2020)

36. Yu, Q., Yan, J.: A novel average dwell time strategy for stability analysis of discret-time switched system by TS fuzzy modeling. Journal of Computcational and Applied Mathematics 391, 113306 (2021)

37. Yu, Y., Zhang, C.: Bifurcation analysis of cascaded H-bridge converter controlled by proportional resonant. Electrical Power and Energy Systems 125, 106476 (2021)

38. Zhang, R., Wu, A., Wang, Z., Cang, S.: Chaotic and subharmonic oscillations in a DC/DC Boost converter with PWM voltage-current hybrid controller and parallel MR load. Nonlinear Dynamics 99, 1321-1339 (2020)

39. Zhu, B., Fan, Q., Li, G., Wang, D.: Chaos suppression for a Buck converter with the memristive load. Analog Integrated Circuits and Signal Processing 107, 309-318 (2021) 